\title{
Resultados do implante bilateral de lente intra-ocular multifocal SA-40N no Hospital de Olhos de Minas Gerais
}

\author{
Visual outcome with binocular implantation of SA-40N multifocal intraocular lens at \\ "Hospital de Olhos de Minas Gerais"
}

\author{
Cristina Moreira Salera ${ }^{1}$ \\ Sérgio Eduardo Marciano Souza² \\ Márcia Reis Guimarães ${ }^{3}$ \\ Ricardo Bicalho Campolina ${ }^{4}$ \\ Ricardo Queiroz Guimarães ${ }^{5}$
}

\begin{tabular}{|c|}
\hline RESUMO \\
\hline 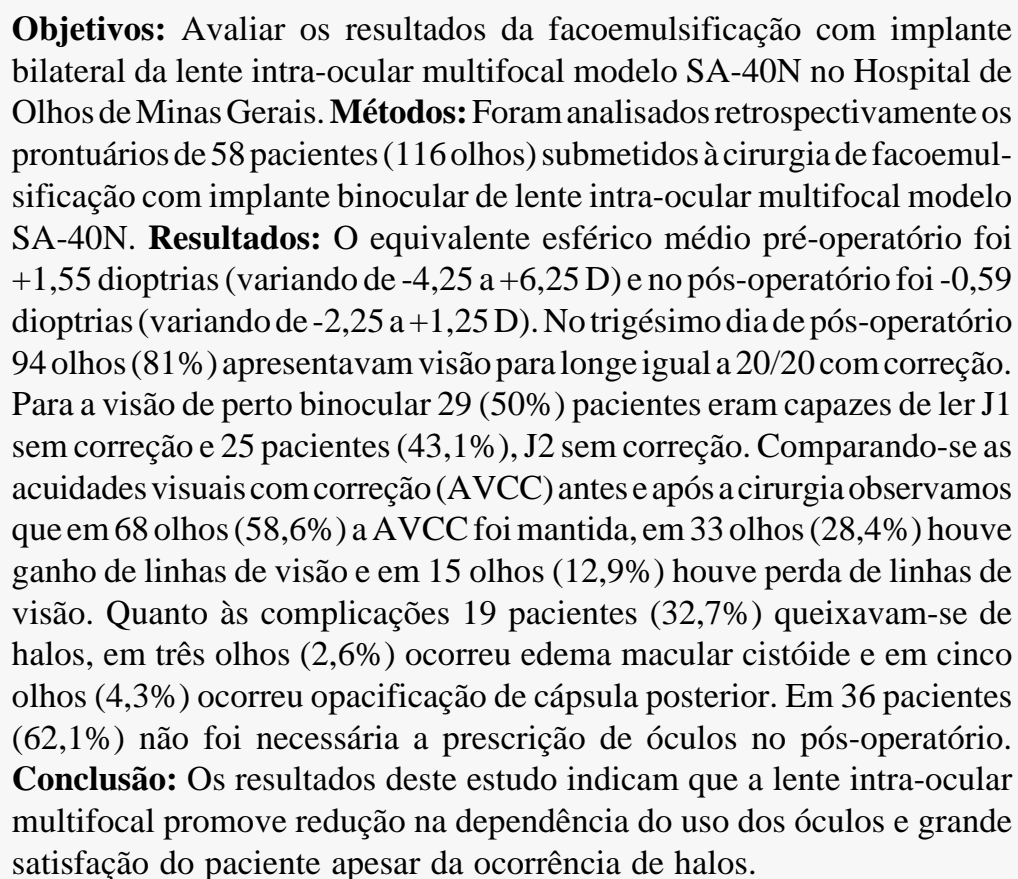 \\
\hline
\end{tabular}

Descritores: Lentes intra-oculares; Refração ocular; Catarata; Implante de lente intraocular; Facoemulsificação

\section{INTRODUÇÃO}

A reabilitação visual ideal após uma cirurgia de catarata seria aquela que proporcionasse uma visão satisfatória para perto e para longe com independência do uso dos óculos ${ }^{(1-6)}$.

$\mathrm{Na}$ ausência da acomodação natural do cristalino, uma alternativa seria o implante da lente intra-ocular (LIO) multifocal ${ }^{(1)}$.

Em 1997, a lente intra-ocular "Allergan Medical Optics (AMO) Array ${ }^{\mathrm{TM}}$ modelo SA-40N tornou-se a única lente intra-ocular multifocal aprovada pelo "FDA" (Food and Drug Administration) para uso nos Estados Unidos ${ }^{(4)}$.

O modelo SA-40N diferencia-se do modelo antecessor (SSM-26NB) apenas pelo índice de refração do silicone utilizado para a confecção da zona óptica que passou a ser 1,46 e pelo material dos hápticos que passou a ser $\mathrm{PMMA}^{(1)}$. No modelo antigo o índice de refração do silicone era 1,41 e os hápticos eram confeccionados com polipropileno ${ }^{(1)}$. 
Esta lente apresenta zonas concêntricas asféricas progressivas em sua superfície anterior com transições menos abruptas entre estas zonas o que proporciona uma redução na percepção dos halos ${ }^{(1)}$.

As zonas 1, 3 e 5 são dominantes para a visão de longe e as zonas 2 e 4 são dominantes para a visão de perto.

No presente estudo analisamos a refração, a visão para perto e para longe, as complicações, o uso de óculos no pósoperatório e a satisfação do paciente com o resultado cirúrgico.

\section{MÉTODOS}

Foram avaliados, retrospectivamente, os prontuários de 58 pacientes (116 olhos) submetidos à facoemulsificação com implante de LIO dobrável multifocal modelo SA-40N (AMO). Além disso, foi realizado questionário subjetivo para a avaliação da satisfação dos pacientes.

As cirurgias foram realizadas por dois cirurgiões (Dr. Ricardo Guimarães e Dr. Ricardo Campolina) no período de novembro de 1998 a abril de 2002.

A avaliação pré-operatória para a cirurgia incluiu o seguinte:

- anamnese (idade, sexo, doenças oculares, doenças sistêmicas, profissão);

- refração dinâmica;

- medida da pressão intra-ocular;

- biomicroscopia;

- fundoscopia;

- microscopia especular (microscópio especular computadorizado sem contato);

- ecobiometria (ecógrafo A com sonda de contato);

- "PAM" (Potencial Acuity Meter).

O cálculo da lente foi realizado objetivando-se uma refração pós-operatória que variava de $-1,00$ a $+0,50$ dioptrias.

A rotina para preparação do paciente para a cirurgia consistiu em sedação com midazolan (Dormonid ${ }^{\circledR}$ ) $3 \mathrm{ml}$ intravenoso e cloridrato de fentanil $0,5 \mathrm{mg}\left(\right.$ Fentanil $\left.^{\circledR}\right) 1 \mathrm{ml}$ intravenoso. Para dilatação da pupila utilizou-se uma gota dos seguintes colírios: cloridrato de proximetacaína $\left(\right.$ Anestalcon $\left.{ }^{\circledR}\right)$, tropicamida (Mydriacyl $\left.{ }^{\circledR}\right)$, cloridrato de fenilefrina (Fenilefrina ${ }^{\circledR}$ ) e flurbiprofeno (Ocufen ${ }^{\circledR}$ ). Em todos os pacientes as cirurgias foram realizadas com bloqueio peribulbar utilizando-se $6 \mathrm{ml} \mathrm{de}$ cloridrato de lidocaína $2 \%$ com vasoconstrictor (1: 400.000).

Quanto à técnica cirúrgica da facoemulsificação, foram realizadas, inicialmente, duas incisões auxiliares ("side port") afastadas $180^{\circ}$ uma da outra e a $90^{\circ}$ da incisão principal que foi realizada no lado temporal ou no meridiano mais curvo da córnea com o objetivo de reduzir o astigmatismo. A seguir a capsulorrexis foi realizada com cistítimo sob irrigação controlada seguida pela realização da incisão principal "clear cornea" cuja extensão padrão era de $3,0 \mathrm{~mm}$ podendo ser ampliada para 3,2 a 3,5 mm quando se objetivava o aplanamento corneano para correção ou diminuição do astigmatismo. Posteriormente realizou-se a hidrodissecção, a facoemulsificação e a aspiração do córtex residual. O polimento da cápsula posterior foi realizado manualmente e foi utilizado um mantenedor de câmara com irrigação contínua de BSS. Após a inje- ção de viscoelástico, a LIO multifocal e foi inserida no saco capsular com o auxílio de pinça. Então, o resíduo de viscoelástico foi removido através do sistema de irrigação e aspiração automatizada. Para finalizar, a câmara anterior foi preenchida com solução salina e, quando necessário, foi realizada hidratação do estroma corneano adjacente às incisões ou sutura com mononylon 10-0.

O paciente foi examinado no dia seguinte e após 30 dias de cirurgia para avaliação refracional sendo a acuidade visual para perto foi testada a 33 centímetros. No período pós-operatório foi prescrito para uso tópico a associação antibiótico/ corticóide (sulfato de neomicina/sualfato de polimixina B e dexametasona) inicialmente quatro vezes ao dia com redução de uma gota a cada semana durante um mês.

\section{RESULTADOS}

Dos 58 pacientes estudados, $38(65,5 \%)$ eram do sexo feminino e 20 (34,5\%) do sexo masculino. A idade variou de 29 a 75 anos (média de 56,1 anos).

$\mathrm{O}$ intervalo entre as cirurgias dos dois olhos variou de 2 a 19 dias (média de 4,2 dias).

A tabela 1 mostra a distribuição da profissão dos pacientes.

$\mathrm{O}$ equivalente esférico pré-operatório médio foi de $+1,55$ dioptrias (variando de $-4,25 \mathrm{a}+6,25 \mathrm{D}$ ) enquanto que o equivalente esférico pós-operatório médio foi de -0,59 dioptrias (variando de $-2,25 \mathrm{a}+1,25 \mathrm{D}$ ) (Gráfico1).

Quando avaliamos a visão para longe sem correção, aos 30

\begin{tabular}{|lc|}
\hline \multicolumn{2}{|c|}{ Tabela 1. Distribuição da profissão dos pacientes } \\
Profissão & Número de pacientes (\%) \\
Aposentado & $15(25,9 \%)$ \\
Do lar & $9(15,5 \%)$ \\
Comerciante & $8(13,8 \%)$ \\
Empresário & $4(6,9 \%)$ \\
Advogado & $4(6,9 \%)$ \\
Professor & $4(6,9 \%)$ \\
Outros & $14(24,1 \%)$ \\
Total & $58(100 \%)$ \\
\hline
\end{tabular}

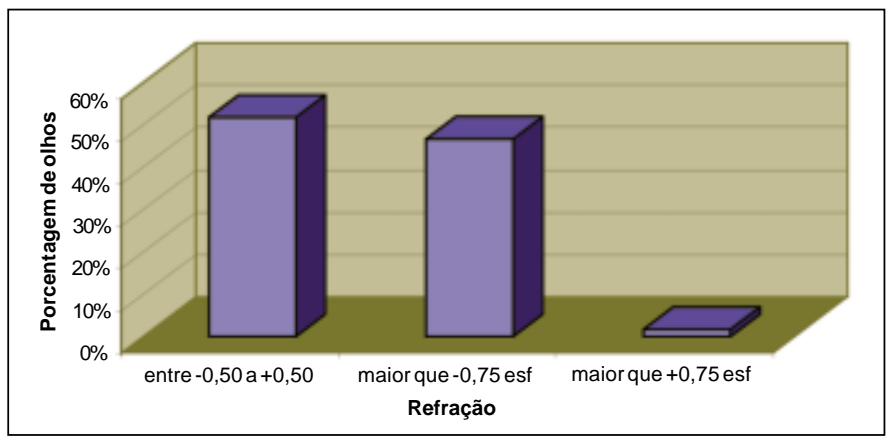

Gráfico 1 - Refração pós-operatória 
dias de pós-operatório, notamos que 78 olhos (67,3\%) apresentavam acuidade visual entre $20 / 20$ e 20/30. Já a acuidade visual para longe, com correção, ficou entre 20/20 e 20/30, em 110 olhos $(94,8 \%)$ (Gráficos 2 e 3).

Ao avaliarmos a visão binocular sem correção para perto observamos que 29 pacientes $(50 \%)$ eram capazes de ler J1 e 25 (43,1\%) liam J2 (Gráfico 4).

Ao compararmos a visão para longe com correção, antes e após a cirurgia, notamos que 68 olhos $(58,6 \%)$ mantiveram a mesma acuidade visual, 23 olhos $(19,8 \%)$ ganharam uma linha de visão e 10 olhos $(8,6 \%)$ ganharam duas ou mais linhas de visão (Gráfico 3). Também foi observado que quatro olhos $(3,4 \%)$ perderam uma linha de visão, seis olhos $(5,2 \%)$ perderam duas linhas de visão e cinco olhos $(4,3 \%)$ perderam três ou mais linhas de visão. Durante o exame realizado com 30 dias de pós-

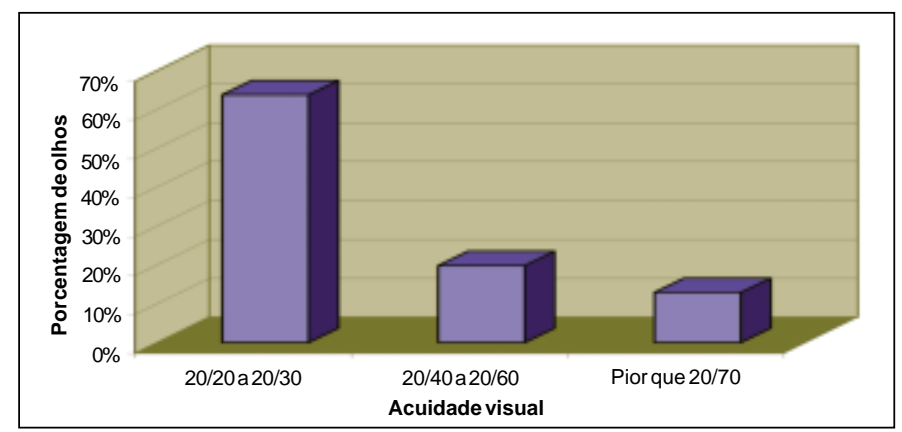

Gráfico 2 - Visão para longe monocular e sem correção

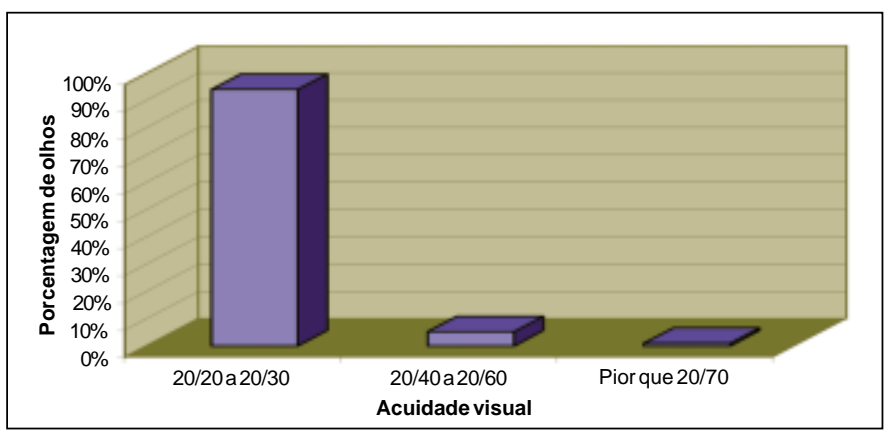

Gráfico 3 - Acuidade visual final com correção

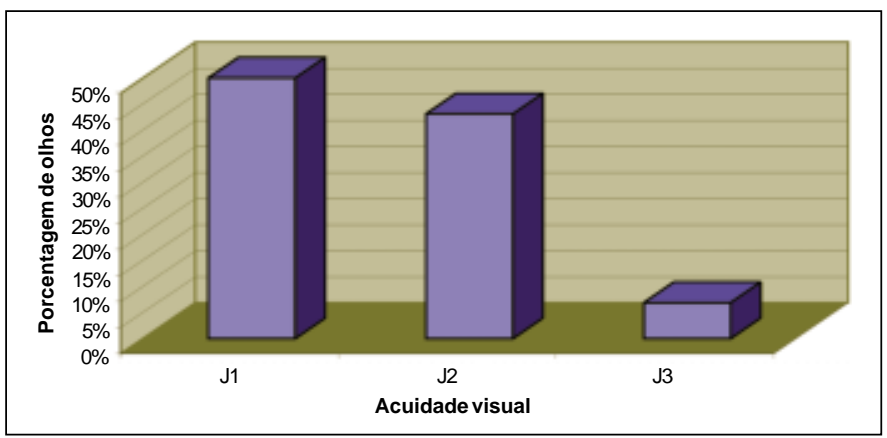

Gráfico 4 - Visão para perto binocular sem correção operatório, observou-se perdas de linhas de visão que ocorreram devido à presença de edema macular cistóide, opacificação de cápsula posterior, uveíte asséptica e percepção de halos.

A LIO multifocal foi programada para diferentes refrações no pós-operatório e obtivemos os melhores resultados quando a programação era para uma refração de $+0,50$ dioptrias (Tabela 2).

No que diz respeito às complicações pós-operatórias, 19 pacientes $(32,7 \%)$ tiveram queixa de halos, em cinco olhos $(4,3 \%)$ houve opacificação da cápsula posterior e em três olhos $(2,6 \%)$ ocorreu edema macular cistóide. Em sete olhos $(6 \%)$ foi realizado LASIK para correção de erro refratométrico residual.

Em 36 pacientes $(62,1 \%)$ não foi necessária a prescrição de óculos e em 17 pacientes $(29,3 \%)$ foi prescrito somente óculos para perto (Gráfico 5).

Foi perguntado aos pacientes o seu grau de satisfação com o resultado cirúrgico e 37 pacientes $(63,8 \%)$ se consideravam satisfeitos como mostra o gráfico 6 .

\begin{tabular}{|lcc|}
\hline \multicolumn{2}{|c|}{ Tabela 2. Programação das LIO's e seus resultados } \\
Programa da LIO & $\begin{array}{c}\text { Número de } \\
\text { olhos (\%) }\end{array}$ & $\begin{array}{c}\text { EE médio } \\
\text { pós-operatório }\end{array}$ \\
$+0,50$ & $52(44,8 \%)$ & $-0,23 \mathrm{D}$ \\
$-0,50$ & $32(27,6 \%)$ & $-0,72 \mathrm{D}$ \\
plano & $22(19,0 \%)$ & $-1,02 \mathrm{D}$ \\
$-1,00$ & $10(8,6 \%)$ & $-0,90 \mathrm{D}$ \\
EE: equivalente esférico & & \\
\hline
\end{tabular}

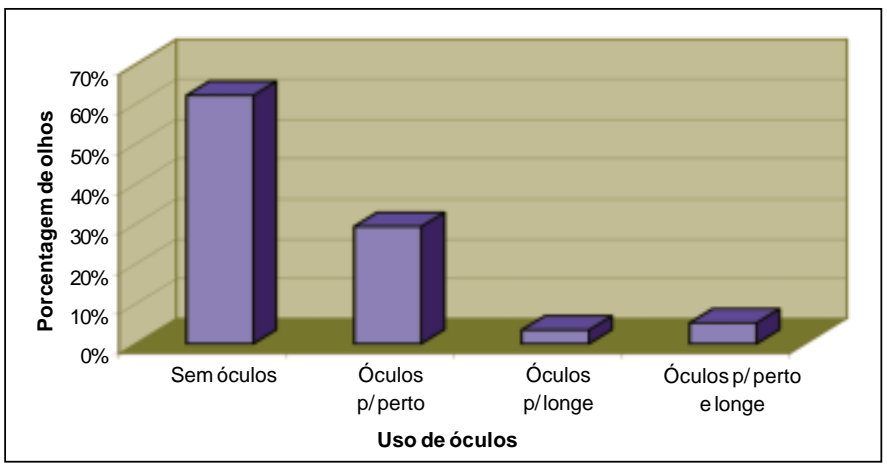

Gráfico 5 - Uso de óculos no pós-operatório

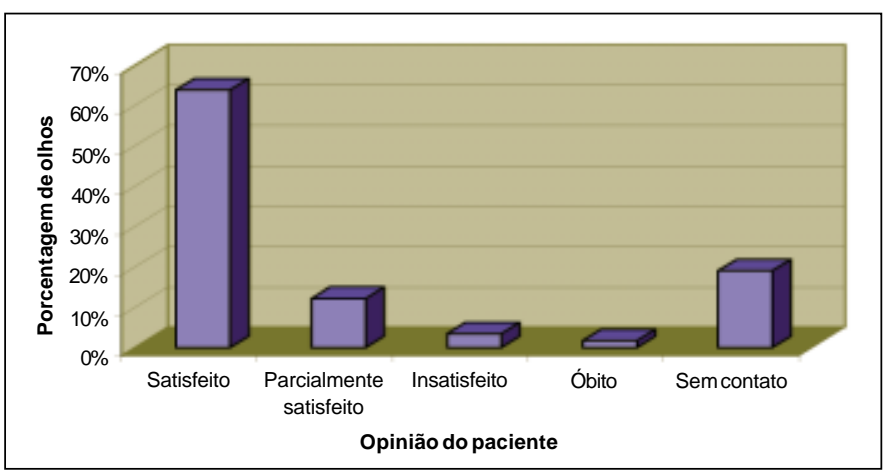

Gráfico 6 - Pesquisa 


\section{DISCUSSÃO}

Atualmente, a cirurgia de catarata padrão é realizada através de uma pequena incisão, seguida pela implantação de lente intra-ocular dobrável. Na maioria dos casos, monofocal ${ }^{(1-2,4,7)}$.

Esse tipo de lente apresenta uma amplitude de foco limitada. Como o olho pseudofácico não é capaz de acomodar, a maioria dos pacientes irá necessitar de óculos para uma adequada visão de perto $^{(1-2,4,7)}$.

No nosso trabalho, ao observarmos a distribuição das profissões dos pacientes, notamos que $25,9 \%$ deles eram aposentados. Esta questão é um fator de extrema importância, uma vez que o sucesso da implantação da lente multifocal encontra-se diretamente relacionado a uma adequada seleção do paciente. A princípio, a lente multifocal é desaconselhada para aqueles pacientes com exigência de visão noturna, uma vez que os halos tornam-se mais intensos à noite, em função do aumento do diâmetro pupilar.

Também é interessante ressaltar que o cirurgião deve sempre advertir quanto às possibilidades e limitações para aqueles pacientes que esperam por "super resultados" e expor as expectativas de forma real.

De acordo com a literatura mundial, a lente intra-ocular multifocal tem proporcionado uma acuidade visual para longe, corrigida ou não, comparável às lentes monofocais. Quanto à visão de perto sem correção, a lente multifocal tem mostrado melhores resultados ${ }^{(1,3,5,7-9)}$.

No presente estudo, $51,7 \%$ dos pacientes ficaram com equivalente esférico pós-operatório entre -0,50 e +0,50 esférico. Resultado semelhante foi encontrado por Jacobi et cols ${ }^{(7)}$. Em $67,3 \%$ dos olhos a acuidade visual monocular, sem correção para longe, ficou entre $20 / 20$ e 20/30. Semelhante aos outros relatos da literatura ${ }^{(1,4,7)}$.

No que diz respeito à visão binocular para perto, sem correção, o nosso estudo também mostrou resultados semelhantes aos de outros autores. Cerca de $50 \%$ dos pacientes eram capazes de ler $\mathrm{J} 1$ e $43,1 \%, \mathrm{~J} 2^{(1,4-5)}$.

Quanto ao cálculo da lente intra-ocular multifocal, obtivemos um equivalente esférico médio pós-operatório mais próximo de plano quando programávamos uma refração final de $+0,50 \mathrm{D}$. De acordo com alguns relatos na literatura mundial, deixar o paciente emétrope ou levemente hipermétrope minimiza a percepção de halos em comparação aos pacientes míopes ${ }^{(2,10-11)}$.

No nosso estudo, 19 (32,7\%) pacientes queixaram-se de halos no pós-operatório o que é uma porcentagem superior ao relatado no estudo de Häring et $\mathrm{al}^{(8)}$. Esta queixa tem-se mostrado mais freqüente naqueles pacientes com lente multifocal do que com a monofocal ${ }^{(8,10)}$. É interessante ressaltar que dos 38 olhos dos 19 pacientes que se queixavam de halos, 26 $(68,4 \%)$ apresentavam miopia na refração final com equivalente esférico médio de-0,89D.

As complicações pós-operatórias que encontramos foram as seguintes: opacificação capsular posterior, uveíte e edema macular cistóide que foram devidamente tratados e apresentaram boa evolução com acuidade visual corrigida final de 20/30 ou melhor.
Não foi necessário o uso de óculos após a cirurgia em 36 $(62,1 \%)$ pacientes o que também foi observado em outros relatos $^{(1,2,4-5,7)}$.

$\mathrm{E}$, de acordo com a informação dos pacientes operados, 37 $(63,8 \%)$ se julgam satisfeitos com o resultado da cirurgia.

\section{CONCLUSÃO}

Nosso estudo mostrou, assim como na literatura mundial, que o resultado do implante da lente intra-ocular multifocal é um ganho na qualidade de vida das pessoas uma vez que proporciona uma menor dependência do uso dos óculos e uma menor limitação nas atividades que requerem a visão de perto.

\section{ABSTRACT}

Purpose: To evaluate the outcomes of phacoemulsification with binocular implantation of SA-40N multifocal intraocular lens at the "Hospital de Olhos de Minas Gerais". Methods: The charts of 58 patients (116 eyes) who were submitted to phacoemulsification with binocular implantation of SA-40N multifocal intraocular lens were retrospectively analyzed. Results: The mean preoperative spherical equivalent was $+1.55 \mathrm{D}$ (ranging from -4.25 to $+6.25 \mathrm{D}$ ) and after surgery it was $-0.59 \mathrm{D}$ (ranging from -2.25 to $+1.25 \mathrm{D}$ ). Thirty days after surgery, 94 eyes $(81 \%)$ achieved 20/20 for better distance corrected visual acuity. For best uncorrected binocular near vision, 29 (50\%) patients were able to read $\mathrm{J} 1$ and $25(43.1 \%)$, J2. When we compare the best corrected distance vision, before and after surgery, we observed that $68(58.6 \%)$ eyes keep the same best corrected visual acuity, $33(28.4 \%)$ eyes gained lines of visual acuity and $15(12.9 \%)$ lost lines of vision. Regarding complications, $19(32.7 \%)$ reported halos, three $(2.6 \%)$ eyes had macular edema and five $(4.3 \%)$ eyes had posterior capsular opacification. Thirty-six patients $(62.1 \%)$ did not need to use spectacles. Conclusion: The results of this study indicate that multifocal intraocular lens bring about less dependence on spectacle wear and high patient satisfaction despite some reports of halos.

Keywords: Lenses, intraocular; Ocular, refraction; Cataract; Lens implantation, intraocular; Phacoemulsification

\section{REFERÊNCIAS}

1. Steinert RF, Aker BL, Trentacost DJ, Smith PJ, Tarantino N. A prospective comparative study of the AMO Array zonal-progressive multifocal silicone intraocular lens and a monofocal intraocular lens. J Cataract Refract Surg. 1999;106(7):1243-55. Commented in: Ophthalmology. 2000;107(10):1801.

2. Sedgewick JH, Orillac R, Link C. Array multifocal intraocular lens in a charity hospital training program - a resident's experience. J Cataract Refract Surg. 2002;28(7):1205 - 10. Commented in: J Cataract Refract Surg. 2003;29(5):858-9.

3. Kamath GG, Prasad S, Danson A, Phillips RP. Visual outcome with the array multifocal intraocular lens in patients with concurrent eye disease. J Cataract Refract Surg. 2000;26(4):576-81. 
4. Javitt JC, Steinert RF. Cataract extraction with multifocal intraocular lens implantation: a multinational clinical trial evaluating clinical, functional, and quality-of-life outcomes. Ophthalmology. 2000;107(11):2040-8.

5. Brydon KW, Tokarewicz AC, Nichols BD. AMO Array multifocal lens versus monofocal correction in cataract surgery. J Cataract Refract Surg. 2000;26(1):96-100.

6. Shoji N, Shimizu K. Binocular function of the patient with the refractive multifocal intraocular lens. J Cataract Refract Surg. 2002;28(6):1012-22.

7. Jacobi PC, Dietlein TS, Luke C, Jacobi FK. Multifocal intraocular lens implantation in presbyopic patients with unilateral cataract. Ophthalmology. 2002;109(4):680-6.

8. Haring G, Dick B, Krummenauer F, Weissmantel U, Kroncke W. Subjective photic phenomena with refractive multifocal and monofocal intraocular lenses. Results of a multicenter questionnaire. J Cataract Refract Surg. 2001;27(2): 245-9. Commented in: J Cataract Refract Surg. 2001;27(7):1148.

9. Akaishi L, Araújo AG, Santos RC, Santos PM. Acuidade visual em implantes bilaterais de lentes intra-oculares monofocais e multifocais. Arq Bras Oftalmol. 2003;66(2):199-205.

10. Hunkeler JD, Coffman TM, Paugh J, Long A, Smith P, Tarantino N. Characterization of visual phenomena with the array multifocal intraocular lens. J Cataract Refract Surg. 2002;28(7):1195-204.

11. Centurion V, Lacava AC, Caballero JC. A performance visual com a lente intraocular multifocal refrativa AMO Array. Rev Bras Oftalmol. 1999;58(5):353-9.

\title{
II CONGRESSO BRASILEIRO DE LENTES DE CONTATO, CÓRNEA E REFRATOMETRIA
}

\section{8 a 30 de Abril de 2005 \\ Castro's Park Hotel - Goiânia - GO}

\author{
Promoção: Sociedade Brasileira de Lentes de Contato, \\ Córnea e Refratometria - SOBLEC \\ Apoio: Conselho Brasileiro de Oftalmologia \\ Sociedade Goiânia de Oftalmologia
}

IN FORMAÇÕES: Consult Eventos

Tel.: (31) 3274-1550

email: consult@ consultcom.com.br

Home-page: www.consulteventos.com.br/ soblec 